\title{
Proteomic Profiling of the Human Tissue and Biological Fluid Proteome
}

Ashley Di Meo ${ }^{1}$, Dorsa Sohaei ${ }^{1,2}$, Ihor Batruch ${ }^{1}$, Pantelis Alexandrou ${ }^{3}$ Ioannis Prassas ${ }^{1,4}$ and Eleftherios P. Diamandis*1,2,4,5

1. Lunenfeld-Tanenbaum Research Institute, Mount Sinai Hospital, Toronto, Canada

2. Department of Laboratory Medicine and Pathobiology, University of Toronto, Toronto, Canada

3. Department of Forensic Medicine and Toxicology, School of Medicine, University of Athens, Greece

4. Department of Pathology and Laboratory Medicine, Mount Sinai Hospital, Toronto, Canada

5. Department of Clinical Biochemistry, University Health Network, Toronto, Canada

Supplemental Figure 1. A, Number of proteins quantitated in each tissue. B, Sequence coverage (\%) distribution across different numbers of proteins. C, Unsupervised hierarchical clustering of protein expression across the analysed tissues. n.d., not determined. D, Distribution of the number of proteins quantified across different numbers of tissues.

Supplemental Figure 2. Venn diagram of the 6,092 unique proteins identified across four brain regions.

Supplemental Figure 3. A, scatterplot of the molecular function GO terms. Color indicates the user-provided p-value; the blue and green circles represent GO terms with more significant $p$-values than the orange and red circles. The GO terms with higher semantic similarity are closer on the plot. B, Network of the molecular function GO terms. Color indicates the user-provided p-value; dark red circles represent GO terms with more significant $\mathrm{p}$-values than light red circles. Highly similar GO terms are linked by edges in the graph, where the line width indicates the degree of similarity.

Supplemental Figure 4. A, scatterplot of the cellular component GO terms. Color indicates the user-provided p-value; the blue and green circles represent GO terms with more significant $\mathrm{p}$-values than the orange and red circles. The GO terms with higher semantic similarity are closer on the plot. B, Network of the cellular component GO terms. Color indicates the user-provided p-value; dark red circles represent GO terms with more significant $\mathrm{p}$-values than light red circles. Highly similar GO terms are linked by edges in the graph, where the line width indicates the degree of similarity.

Supplemental Table 1. Overview of the 46 normal human tissues from five adult donors collected post-mortem at autopsy. 


\section{Supplemental Figure 2}

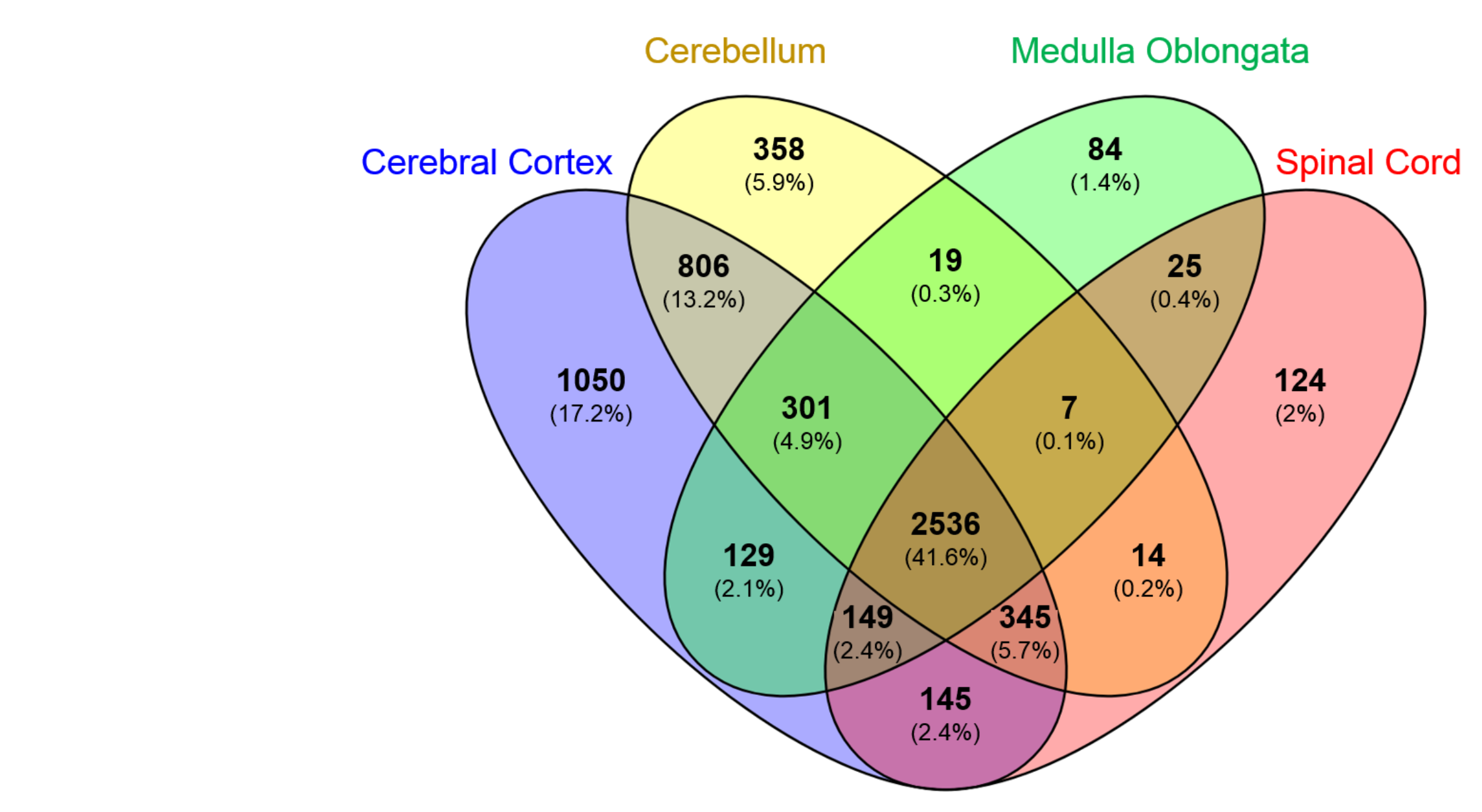




\section{Supplemental Figure 3}

A

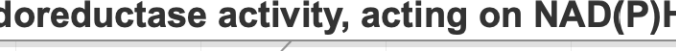

6- peroxidase activity

glutathione binding

rotein dimerization activity

antioxidant activity

structural molecule activity

ion channel binding

catalytic activity

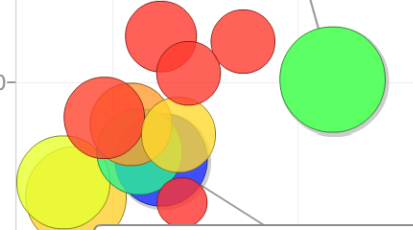

oxidoreductase activity

MHC class II protein complex binding

$$
\text { oxygen transporter activity }
$$

acetyl-CoA C-acyltransferase activity

7
0
0
0
0
0
0
0
$\frac{0}{0}$
$\frac{0}{0}$
0
0

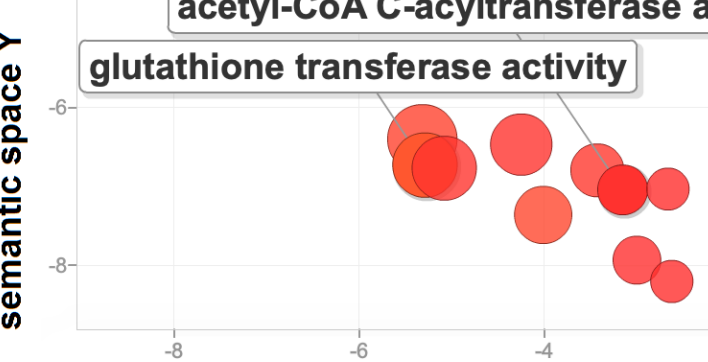

semantic space $X$ cadherin binding myosin binding

molecule binding electron carrier activity
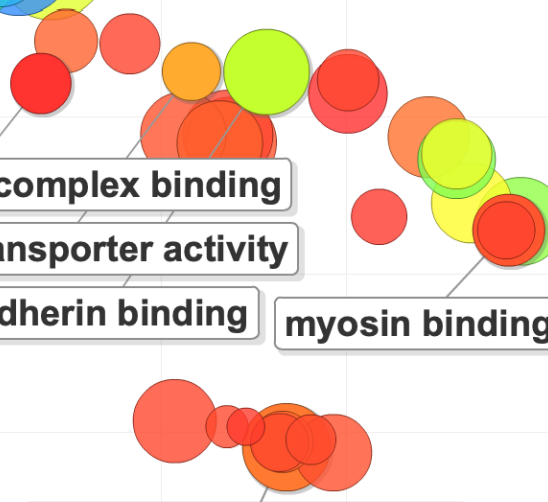

enzyme inhibitor activity

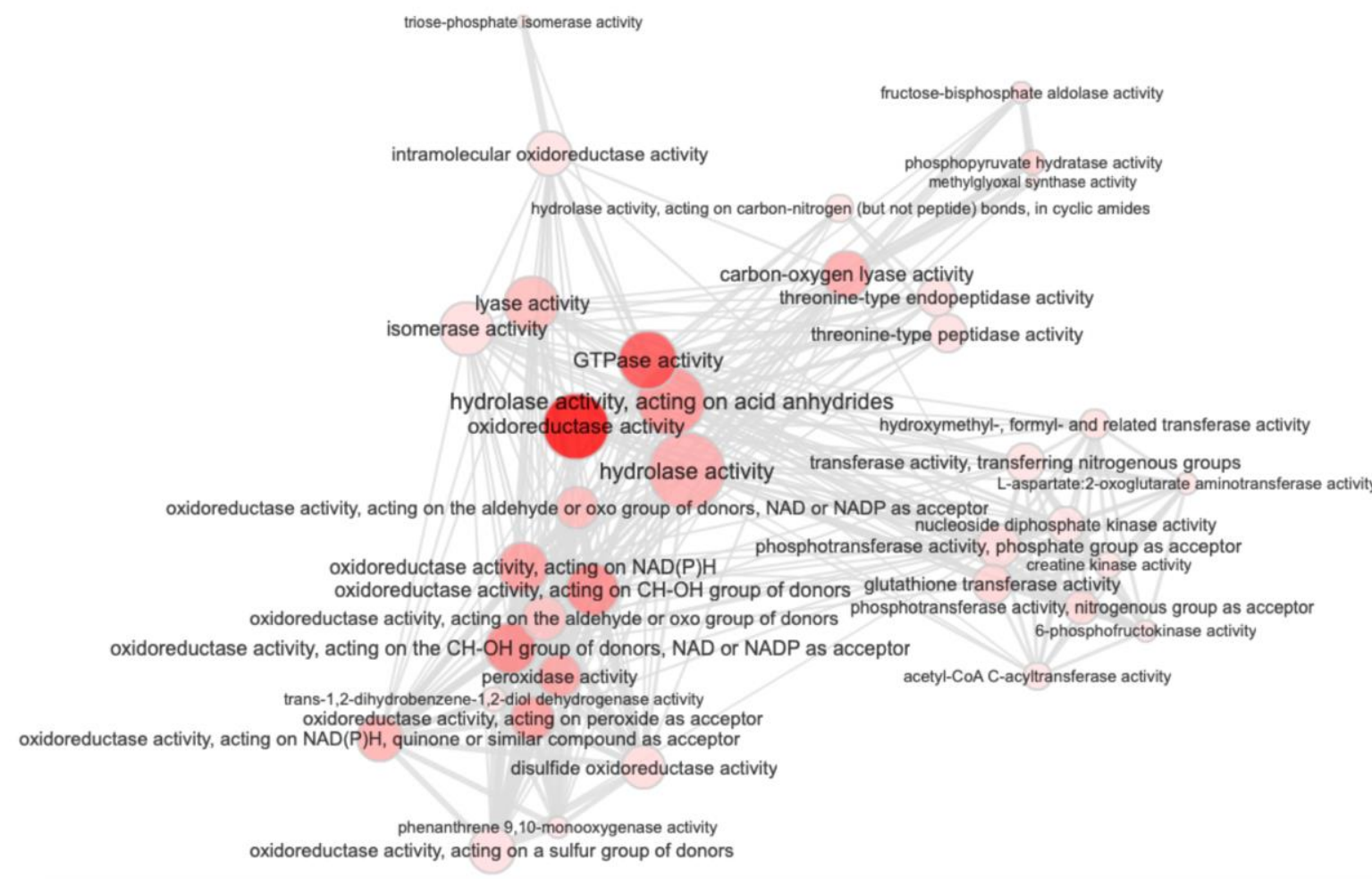




\section{Supplemental Figure 4}

A
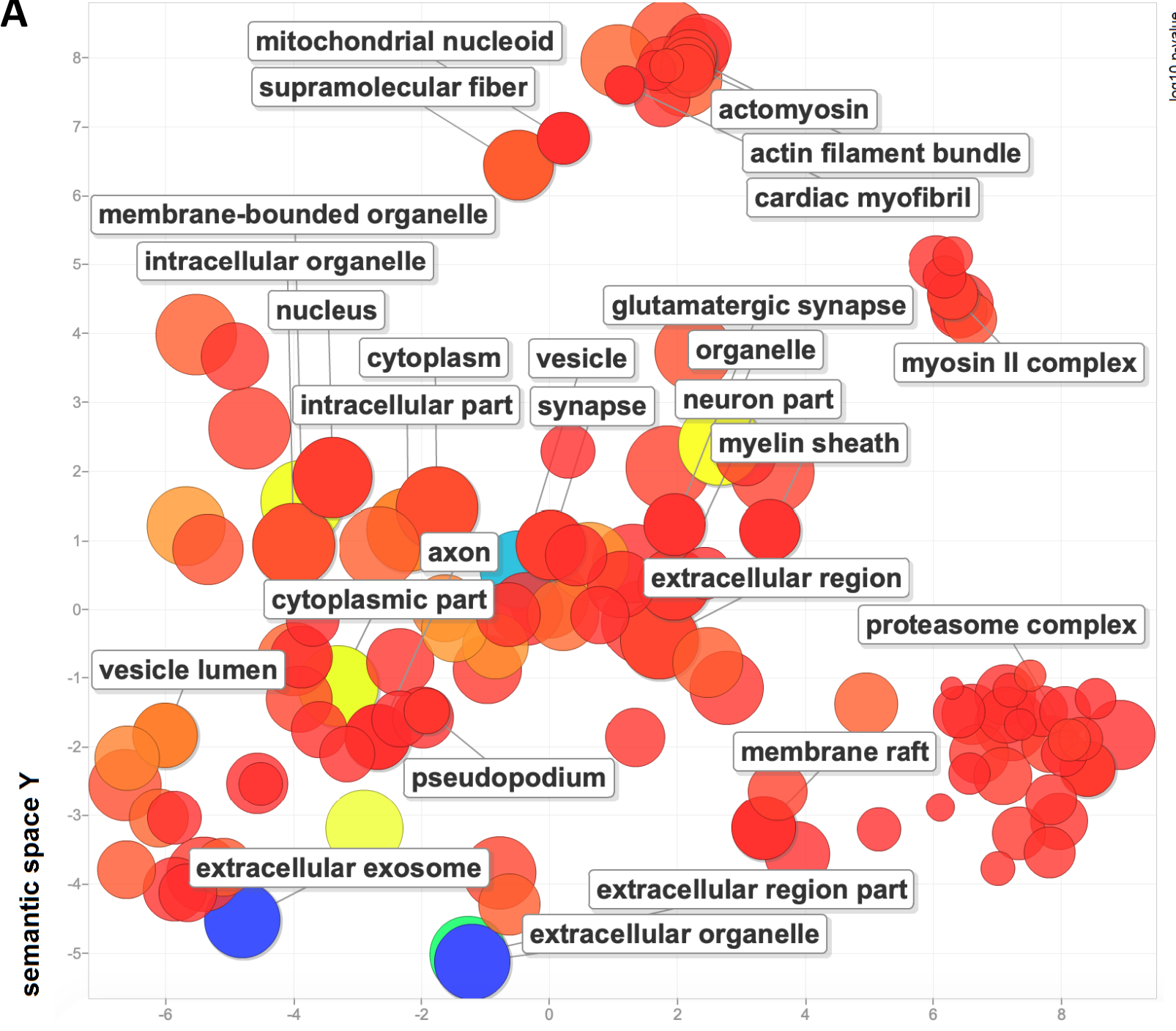

semantic space $X$

actomyosin

actin filament bundle

cardiac myofibril

glutamatergic synapse

vesicle organelle myosin II complex

myelin sheath

extracellular region

membrane raft
B

pigment granule

xtracellular space

chylomicron

zona pellucida receptor complex

endoplasmic reticulum lumen

77

s.

COP9 signalosome
supramolecular fiber F-actin capping protein complex respiratory chain complex

cytoplasmic vesicle cytoskeleton

$\begin{array}{lll}\text { nucleosome } & \\ \text { myosin Il complex }\end{array}$ intracellular organelle lumen catalytic complex

intermediate filament cytoskeleton
myosin complex

$\begin{array}{ll}\text { actin filament } & \text { Arp2/3 protein complex } \\ \text { mitochondrial nucleoid } & \end{array}$

organelle inner membranese complex proteasome complex mitochondrial matrix actin cytoskeleton cardiac myofibril

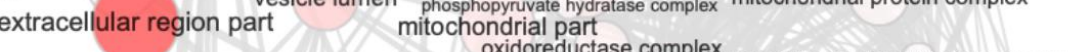

1. peptidase complex actomyosin endoplasmic relculum chaperone complex

intracellular organelle part
extracellular exosome

ribonucleoprotein complex
chaperonin-containing T-complex

mitochondrial intermembrane space
ficolin-1-1-ich granule lumen

pigment granule
tertiary granule lumen
melanosome

chylomicron extracellular organelle

endocytic vesicle

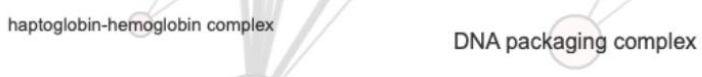

cytoplasmic vesicle part
DNA packaging complex 


\section{Supplemental Table 1}

\begin{tabular}{|c|c|c|c|c|c|c|}
\hline Tissue & Patient 1 & Patient 2 & Patient 3 & Patient 4 & Patient 5 & TOTAL \\
\hline Skin & & & & & & 3 \\
\hline Ovary & & & & & & 5 \\
\hline Adrenal Gland & & & & & & 5 \\
\hline Appendix & & & & & & 5 \\
\hline Lung & & & & & & 5 \\
\hline Liver & & & & & & 5 \\
\hline Heart & & & & & & 5 \\
\hline Colon & & & & & & 5 \\
\hline Brain & & & & & & 5 \\
\hline Esophagus & & & & & & 5 \\
\hline Stomach & & & & & & 5 \\
\hline Small intestine & & & & & & 5 \\
\hline Trachea & & & & & & 5 \\
\hline Kidney & & & & & & 4 \\
\hline Cervix & & & & & & 2 \\
\hline Bladder & & & & & & 5 \\
\hline Breast & & & & & & 2 \\
\hline Spleen & & & & & & 5 \\
\hline \begin{tabular}{|l|} 
Duodenum \\
\end{tabular} & & & & & & 4 \\
\hline \begin{tabular}{|l|} 
Uterus \\
\end{tabular} & & & & & & 2 \\
\hline \begin{tabular}{|l} 
Gallbladder \\
\end{tabular} & & & & & & 4 \\
\hline Ureter & & & & & & 2 \\
\hline Thyroid & & & & & & 2 \\
\hline \begin{tabular}{|l} 
Pancreas \\
\end{tabular} & & & & & & 2 \\
\hline Adipose tissue & & & & & & 2 \\
\hline Lymph node & & & & & & 2 \\
\hline \begin{tabular}{|l} 
Salivary gland \\
\end{tabular} & & & & & & 2 \\
\hline Skeletal muscle & & & & & & 2 \\
\hline \begin{tabular}{|l|} 
Prostate \\
\end{tabular} & & & & & & 1 \\
\hline Seminal vesicle & & & & & & 1 \\
\hline \begin{tabular}{|l|} 
Testis \\
\end{tabular} & & & & & & 1 \\
\hline Epididymis & & & & & & 1 \\
\hline Anus & & & & & & 1 \\
\hline Bone marrow & & & & & & 1 \\
\hline Fallopian tube & & & & & & 1 \\
\hline \begin{tabular}{|l|lll} 
Medulla oblongata \\
\end{tabular} & & & & & & 1 \\
\hline \begin{tabular}{|l} 
Parathyroid gland \\
\end{tabular} & & & & & & 2 \\
\hline Tonsil & & & & & & 1 \\
\hline \begin{tabular}{|l|} 
Placenta \\
\end{tabular} & & & & & & 1 \\
\hline Endometrium & & & & & & 1 \\
\hline Frontal & & & & & & 1 \\
\hline Parietal & & & & & & 1 \\
\hline Temporal & & & & & & 1 \\
\hline Occipital & & & & & & 1 \\
\hline Cerebellum & & & & & & 1 \\
\hline \begin{tabular}{|l|} 
Spinal Cord \\
\end{tabular} & & & & & & 1 \\
\hline
\end{tabular}

